\title{
Influências dos modos de operação nas emissões de poluentes provenientes de veículos flex em região
}

\section{urbana}

\author{
Julie Anne Holanda Azevedo ${ }^{1}$, Demostenis Ramos Cassiano ${ }^{2}$, Bárbara Brito Feitosa ${ }^{3}$, \\ Mona Lisa Moura de Oliveira ${ }^{4}$, Ed Pinheiro Lima ${ }^{5}$, Bruno Vieira Bertoncini ${ }^{6}$
}

\author{
'Universidade Federal do Ceará, Fortaleza, CE, Brasil, julie@det.ufc.br \\ 2Universidade Federal do Ceará, Fortaleza, CE, Brasil, cassiano@det.ufc.br \\ 3Universidade Federal do Ceará, Fortaleza, CE, Brasil, barbarabrito@det.ufc.br \\ ${ }^{4}$ Universidade Estadual do Ceará, Fortaleza, CE, Brasil, mona.lisa@uece.br \\ 5Universidade Estadual de Maringá, Maringá, PR, Brasil, ed_p_lima@outlook.com \\ 6Universidade Federal do Ceará, Fortaleza, CE, Brasil, bruviber@det.ufc.br
}

\section{Recebido:}

14 de janeiro de 2017

Aceito para publicação:

22 de maio de 2017

Publicado:

30 de agosto de 2017

Editor de área:

Flávio Cunto, UFC

\section{Palavras-chaves:}

Emissões.

Veículos flex.

Modos de operação.

Ciclo de condução.

\section{Keywords:}

Emissions.

Flex-fuel vehicle.

Operating mode.

Driving cycle.

DOI:10.14295/transportes.v25i2.1304

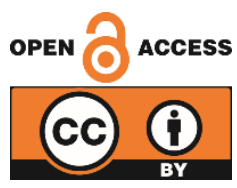

\begin{abstract}
RESUMO
Este trabalho se propõe a verificar a influência dos modos de operação nas emissões de poluentes provenientes de veículos flex. Para isto, um ciclo de condução real foi desenvolvido para o veículo leve tipo flex em um trecho urbano da cidade de Fortaleza. Para a coleta de dados foi utilizado um método on-board de coleta instantânea de parâmetros cinemáticos, de motor, de emissões veicular e de via. Devido às características locais do tráfego, observou-se uma maior representatividade do modo de operação idle. Em geral, observou-se ainda uma maior influência do modo de operação aceleração nas emissões de poluentes e no consumo de combustíveis. A maior taxa de emissão média de $\mathrm{CO}_{2}$ foi observada durante o modo de operação aceleração $(6,38 \mathrm{mg} / \mathrm{s})$, seguido do modo idle $(4,70$ $\mathrm{mg} / \mathrm{s})$. Para o poluente $\mathrm{CO}$, a maior taxa de emissão média foi observada também durante o modo de operação aceleração $(55,60 \mu \mathrm{g} / \mathrm{s})$, seguido do modo idle $(35,01 \mu \mathrm{g} / \mathrm{s})$. Já para o poluente $\mathrm{NO}_{x}$, maiores taxas de emissão foram observadas no modo de operação acelera-

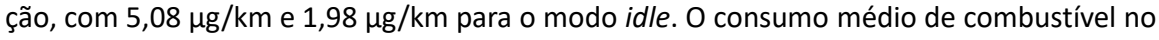
modo de operação aceleração foi de $6,61 \mathrm{~L} / 100 \mathrm{~km}$ durante os testes, seguido dos modos idle e desaceleração, com 4,84 e 3,39 L/100 km, respectivamente.
\end{abstract}

\section{ABSTRACT}

This work proposes to verify the influence of operating modes on pollutants emissions of flex - fuel vehicles. For this, a real driving cycle was developed for the flex light vehicle in an urban street of the Fortaleza city. For data collection was used a method of instant collection of kinematic parameters from engine, road and vehicle emissions. Due to the local characteristics of the traffic, a greater representatively of the idle operating modes was observed. In general, there was a greater influence of the acceleration mode on pollutant emissions and fuel consumption. The highest average $\mathrm{CO}_{2}$ emission rate was observed during acceleration mode $(6.38 \mathrm{mg} / \mathrm{s})$, followed by the idle mode $(4.70 \mathrm{mg} / \mathrm{s})$. For the $\mathrm{CO}$ pollutant, the highest average emission rate was also observed during the acceleration mode $(55.60 \mu \mathrm{g} / \mathrm{s})$, followed by the idle mode $(35.01 \mu \mathrm{g} / \mathrm{s})$. For the $\mathrm{NO}_{\mathrm{x}}$ pollutant, higher emission rates were observed in the acceleration mode, $5.08 \mu \mathrm{g} / \mathrm{km}$ and $1.98 \mu \mathrm{g} / \mathrm{km}$ for the idle mode. The average fuel consumption in the acceleration mode was $6.61 \mathrm{~L} / 100 \mathrm{~km}$ during the tests, followed by idle and deceleration modes, with 4.84 and $3.39 \mathrm{~L} / 100 \mathrm{~km}$, respectively.

\section{INTRODUÇÃO}

O setor de transportes é primordial para o desenvolvimento econômico e social, permitindo a mobilidade de pessoas e uma eficiente logística de recursos. De acordo com o Departamento Nacional de 
Trânsito (DENATRAN), circularam pelas ruas e estradas do Brasil, no ano de 2016, cerca de 89 milhões de veículos automotores.

Entretanto, o aumento dessa motorização individual, consequência da deficiência crônica dos sistemas de transporte de massa, tem intensificado o tráfego nos grandes centros urbanos. Esta intensificação do tráfego provoca, diretamente, um aumento significativo dos congestionamentos e, indiretamente, a degradação ambiental devido à emissão de poluentes e da poluição sonora, resultando na diminuição da qualidade de vida nas cidades.

Com o objetivo de reduzir a emissão de poluentes automotivos, principalmente os níveis de $\mathrm{CO}_{2}$, o governo brasileiro em sua política energética estimula a inclusão de fontes renováveis e determinou, em março de 2015, o teor de $27 \%$ de álcool anidro, derivado da cana de açúcar, na gasolina (Mapa, 2015). Segundo DENATRAN, cerca de 35,87\% dos automóveis circulantes no Brasil são de tecnologia flex (DENATRAN, 2016).

Devido ao modo de operação dos veículos ter impacto significativo nas emissões dos poluentes, vários esforços têm sido realizados por pesquisadores na tentativa de quantificar as emissões veiculares em diversos modos de operação. Neste contexto, a análise das emissões em ciclos de condução se apresenta como um conceito importante na modelagem e estimação das emissões provenientes de veículos automotores (Xiao et al., 2012).

Ciclos de condução são compostos por uma série de modos de operação do veículo, incluindo veículo parado, cruzeiro e em instantes de aceleração e desaceleração, envolvendo distribuições de velocidade durante o trajeto. Assim, modo de operação parado se refere à situação em que tanto a aceleração quanto a velocidade são nulas; cruzeiro quando a velocidade for diferente de zero e a aceleração for nula; aceleração quando essa taxa for positiva e a velocidade for não nula; e desaceleração quando essa taxa for negativa e a velocidade for diferente de zero.

Em virtude dessa situação, Conselho Nacional do Meio Ambiente (CONAMA) o instituiu em 1986 o Programa de Controle de Poluição do Ar por Veículos Automotores (PROCONVE) como forma de controlar a emissão de poluentes de fontes móveis, responsáveis por degradar a qualidade do ar nos centros urbanos, fixando prazos, limites máximos de emissão e estabelecendo exigências tecnológicas para veículos automotores nacionais e importados. O PROCONVE adota a metodologia de medição dos poluentes para veículos leves novos segundo a NBR 6601 - Análise dos Gases de Escapamento de Veículos Rodoviários Automotores Leves à Gasolina, conforme ABNT, 2001, baseada na metodologia da Agência de Proteção Ambiental americana (EPA). Esta norma prescreve o método para a determinação de hidrocarbonetos $\left(\mathrm{HC}\right.$ ), monóxido de carbono (CO), óxidos de nitrogênio $\left(\mathrm{NO}_{\mathrm{x}}\right.$ ) e dióxido de carbono $\left(\mathrm{CO}_{2}\right)$, emitidos pelos motores de combustão internas dos veículos automotores leves. Segundo Abreu e Ribeiro (2003), estas emissões refletem as condições de velocidade média em tráfego urbano de 31,5 $\mathrm{km} / \mathrm{h}$; temperatura ambiente de 20 a $30{ }^{\circ} \mathrm{C}$ e umidade relativa do ar de 40 a $60 \%$, podendo ser usadas sem correções no caso de pequenas variações destas condições, devido a se tratarem de dados estimados.

Esta medida é executada por meio do amostrador volumétrico constante associado à simulação veicular, em um ensaio que representa condições de uso normal médio em trânsito urbano. Esse sistema de amostragem permite a medição das massas reais dos poluentes emitidos pelo motor através do tubo de descarga do veículo e coletadas em bolsas (bag) (ABNT, 2001), sendo que, para cada fase do ciclo é associado uma bag, com as respectivas emissões. No ensaio do ciclo FTP, as condições de trânsito urbano são simuladas por um dinamômetro de chassi, que segue uma curva denominada ciclo de condução FTP-75, onde o motorista segue o ciclo mostrado em um monitor, acelerando e freando o veículo (Vaz de Melo, 2005).

Apesar do amplo uso em testes de ciclo de condução na regulamentação e calibração de modelos de emissão, existem estudos que apontam diferenças entre o que é medido em laboratório e o que ocorre no uso do veículo. Pelkmans e Debal (2006) apontam grandes diferenças entre as emissões e o consumo de um veículo real e o ciclo de condução europeu. Os autores obtiveram resultados que mostraram 
que no teste em ciclo de condução com um veículo com ano de fabricação do ano 2000 pode apresentar valores de emissões de $\mathrm{CO}$ e $\mathrm{NO}_{\mathrm{x}}$ até 10 vezes menores em comparação a situações reais de tráfego, além de subestimar os valores de consumo e emissões de $\mathrm{CO}_{2}$ de 10 a $20 \%$.

Em geral, um ciclo de condução característico do cenário urbano apresenta constantes variações de velocidade devido à influência de particularidades da infraestrutura viária, dos limites de velocidade e da densidade de tráfego. Quando são gerados congestionamentos, aumenta-se a frequência de eventos transitórios como acelerações/desacelerações e de paradas e partidas, que afetam significativamente as taxas de emissões (Cappiello, 2002).

Nesse contexto, o presente estudo objetivou verificar a influência dos modos de operação de veículos flex nas emissões de $\mathrm{CO}_{2}, \mathrm{CO}, \mathrm{NO}_{x}, \mathrm{HC}, \mathrm{SO}_{2}$. Em complementação, foram desenvolvidos os seguintes objetivos específicos: (i) elaborar um ciclo de condução representativo para veículos leves em tráfego urbano; (ii) relacionar as emissões de cada poluente analisado a cada modo de operação; e (iii) verificar a influência de parâmetros dinâmicos de operação do veículo nas emissões de poluentes.

\section{METODOLOGIA ADOTADA}

\subsection{Região de estudo}

A cidade de Fortaleza, capital do Estado do Ceará, está localizada na região Nordeste do Brasil. Segundo dados do Instituto Brasileiro de Geografia e Estatística (IBGE), possui 2.609.716 habitantes, sendo a quinta mais populosa capital do país (IBGE, 2016). A cidade possui ainda a maior frota do Estado, com 1.023.886 veículos (DENATRAN, 2016).

A região de estudo é caracterizada por intenso tráfego veicular, presença de estabelecimentos comerciais, residências e de instituições de ensino, sendo considerada uma das vias mais congestionadas da cidade. 0 percurso foi composto por três grandes avenidas, classificadas como coletora (Avenida Jovita Feitosa) e arteriais tipo I (Avenidas Treze de Maio e Pontes Vieira), como mostra a Figura 1.

(a)

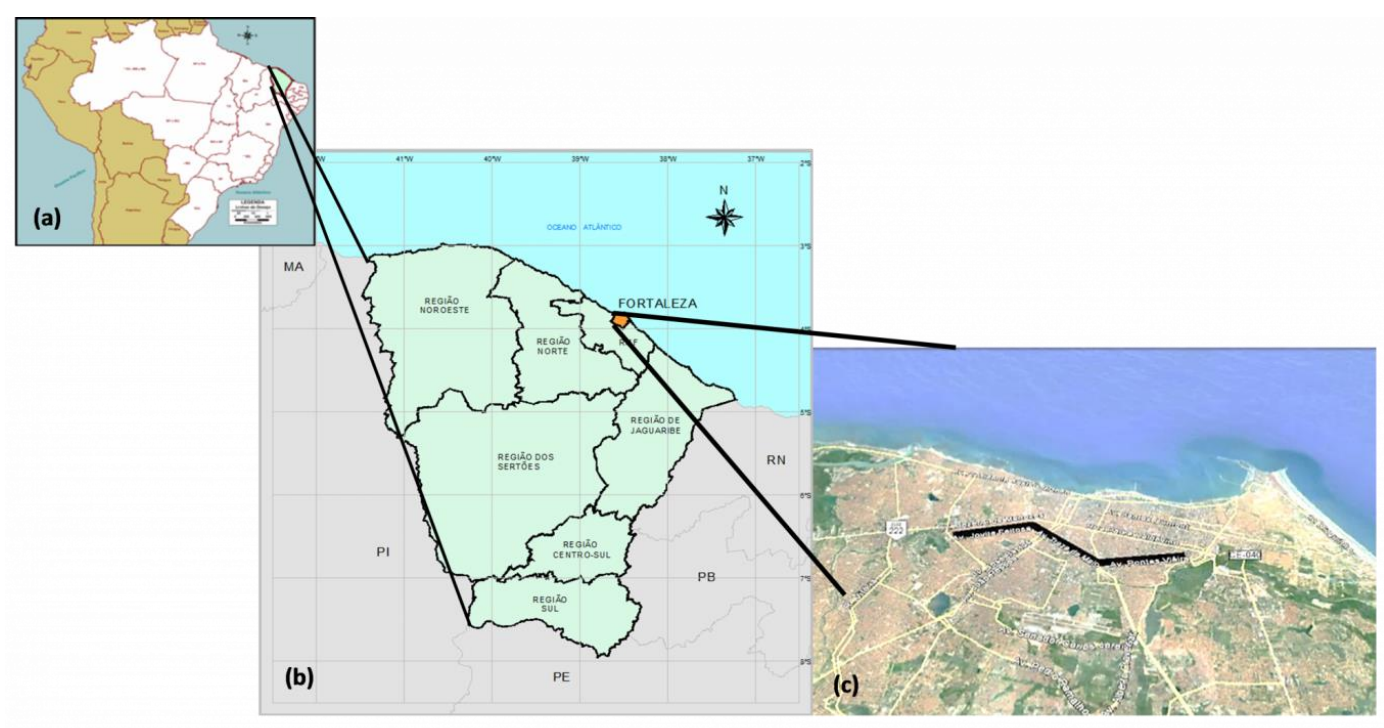

(b)

(c)

Figura 1. (a) Brasil, (b) Estado do Ceará, (c) Percurso do ciclo de condução realizado na cidade de Fortaleza, Ceará,Brasil

\subsection{Veículo teste}

Os ensaios foram realizados em dias típicos com uso de um veículo sedan compacto flex, modelo Nissan Versa 1.6 SV. As especificações estão apresentadas na Tabela 1. 0 combustível utilizado foi adquirido no mercado local, contendo teor de álcool etílico anidro de $27 \%$, determinado em laboratório, conforme NBR 13992 de 2015 e recomendação brasileira através da Portaria 72/2015 (MAPA, 2015). 
Tabela 1: Especificações do veículo utilizado nos testes

\begin{tabular}{ll}
\hline Veículo Padrão & Característica \\
\hline Veículo & Nissan Versa 1.6 SV \\
Ano/Modelo & $2014 / 2014$ \\
Quilometragem & $30,773 \mathrm{~km}$ \\
Combustível & Flex \\
Massa & $1088 \mathrm{~kg}$ \\
Válvulas & 16 \\
Cilindros & 4 \\
Cilindradas & $1.600 \mathrm{cc}$ \\
Potência (gasolina/álcool) & $111 \mathrm{cv} @ 5.600 \mathrm{rpm}$ \\
Sistema de Tratamento & Catalisador de três vias \\
Padrão de emissão & Proconve L6/EURO V \\
Transmissão & Manual, 5 velocidades \\
\hline
\end{tabular}

\subsection{Sistema Portátil para Medição de Emissões}

0 veículo foi equipado com um sistema embarcado portátil para medição de emissões (do inglês Portable Emissions Measurement System) conectado à unidade de controle do motor através da porta de diagnóstico on-board (OBD), permitindo o monitoramento de diversos parâmetros com frequência de $1 \mathrm{~Hz}$, que estão apresentados na Figura 2.

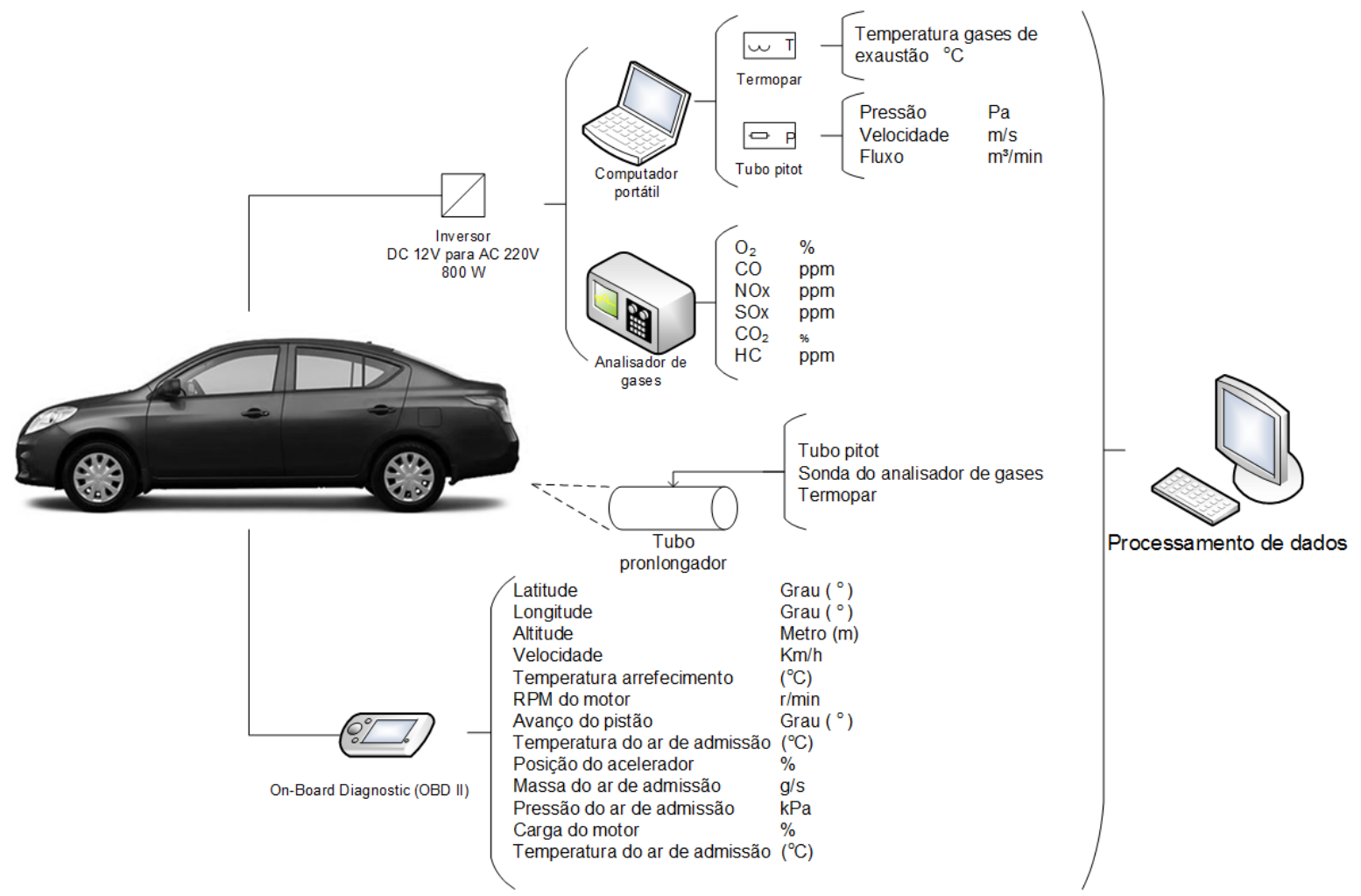

Figura 2. Parâmetros cinemáticos e de emissões monitorados em tempo real (1 Hz)

Altitude, latitude e longitude foram registradas continuamente por um dispositivo GPS. Todos os componentes do sistema de coletas foram previamente calibrados e certificados. 0 consumo de combustível foi calculado pelo método de balanço de carbono, segundo a NBR 7024. Os dados de massa dos pesquisadores e dos equipamentos a bordo foram coletados e considerados no carregamento do veículo. Os ensaios tiveram duração de aproximadamente 11.000 segundos. Maiores detalhes no desenvolvimento deste PEMS foram descritos por Cassiano et al. (2016) e Dias et al. (2015). 


\section{RESULTADOS E DISCUSSÕES}

O ciclo de condução padrão adotado no Brasil, descrito na NBR 6601, é baseado no ciclo do Federal Test Procedure, chamado de FTP-75, metodologia americana desenvolvida em chassi dinamométrico para certificação de emissões de poluentes para veículos leves. 0 ciclo de condução FTP-75 é composto de três fases (Figura 3) o primeiro destinado à análise das emissões na fase transitória com partida a frio (505 s), a segunda, destinada à análise das emissões na fase estabilizada (866 s) e a terceira destinada à análise das emissões na fase transitória com partida a quente (505 s), sendo que entre o fim da segunda fase e o começo da terceira, o motor é desligado por $10 \pm 1$ minutos, totalizando uma viagem de 12,1 km (ABNT, 2001; Cappiello, 2002).

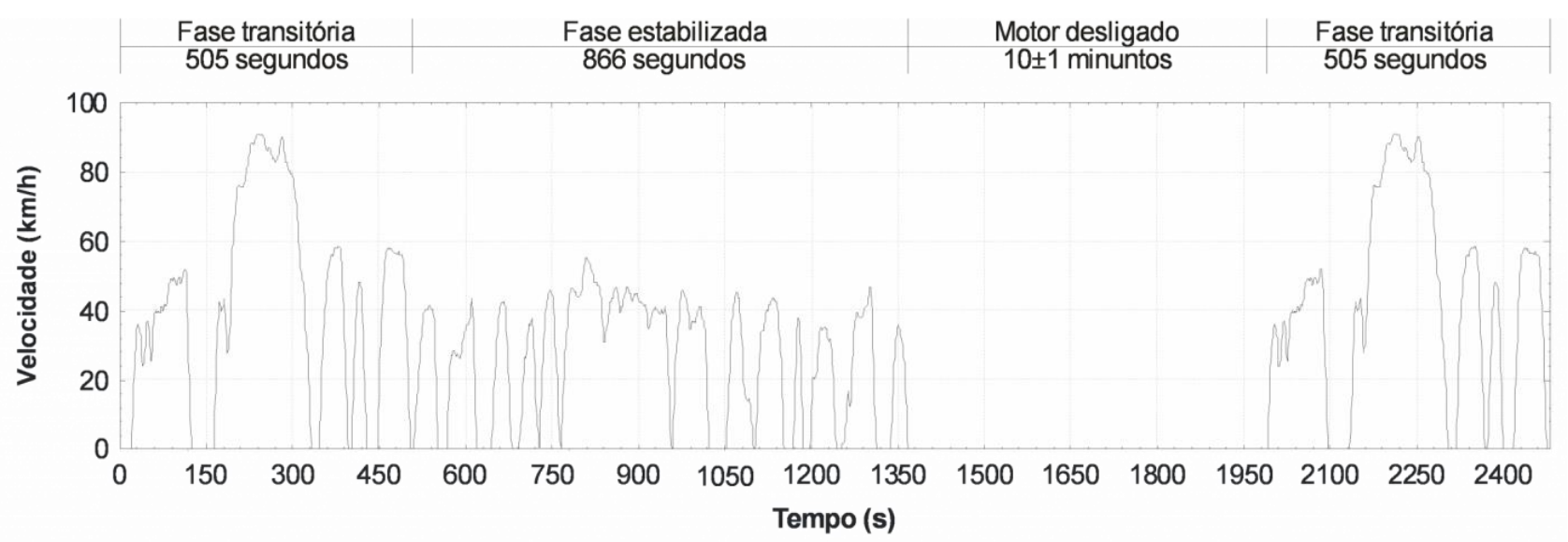

Figura 3. Ciclo de condução FTP-75

A utilização deste ciclo de condução não confere veracidade, por não incorporar diversos parâmetros imprescindíveis, como geometria e inclinação de via, comportamento do condutor e combustível, tornando a adoção do FTP-75 um procedimento não representativo da realidade. Além destes parâmetros, outros como tempo, velocidade, aceleração são consideravelmente diferentes, o que implica em emissões diferentes. Resumidamente, a Tabela 1 apresenta as diferenças observadas entre o ciclo de condução real desenvolvido na cidade de Fortaleza - CE e o ciclo de condução padrão adotado no Brasil FTP-75.

Tabela 1: Diferenças observadas entre o ciclo de condução real desenvolvido na cidade de Fortaleza e o ciclo de condução padrão adotado no Brasil FTP-75

\begin{tabular}{ccc}
\hline Parâmetros & FTP-75 & Real/Fortaleza \\
\hline Distância $(\mathrm{km})$ & 17,8 & 8,4 \\
Tempo $(\mathrm{s})$ & 1874 & 1216 \\
Velocidade média $(\mathrm{km} / \mathrm{h})$ & 34,1 & 23,8 \\
Velocidade máxima $(\mathrm{km} / \mathrm{h})$ & 91,2 & 76,0 \\
Aceleração mínima $\left(\mathrm{m} / \mathrm{s}^{2}\right)$ & $-1,5$ & $-5,6$ \\
Aceleração média $\left(\mathrm{m} / \mathrm{s}^{2}\right)$ & 0,0 & 0,0 \\
Aceleração máxima $\left(\mathrm{m} / \mathrm{s}^{2}\right)$ & 1,5 & 3,1 \\
\hline
\end{tabular}

0 ciclo de condução desenvolvido para a cidade de Fortaleza teve duração de 1.216 s, em que a velocidade máxima atingida durante o percurso foi de $76,0 \mathrm{~km} / \mathrm{h}$ e a velocidade média $23,8 \mathrm{~km} / \mathrm{h}$. 0 ciclo de condução real apresenta, ainda, um perfil de aceleração inerente a variabilidade do tráfego local, variando entre $-5,6 \mathrm{~m} / \mathrm{s}^{2}$ e $3,1 \mathrm{~m} / \mathrm{s}^{2}$. A Figura 4 apresenta o ciclo de condução desenvolvido para o veículo leve na cidade de Fortaleza. 


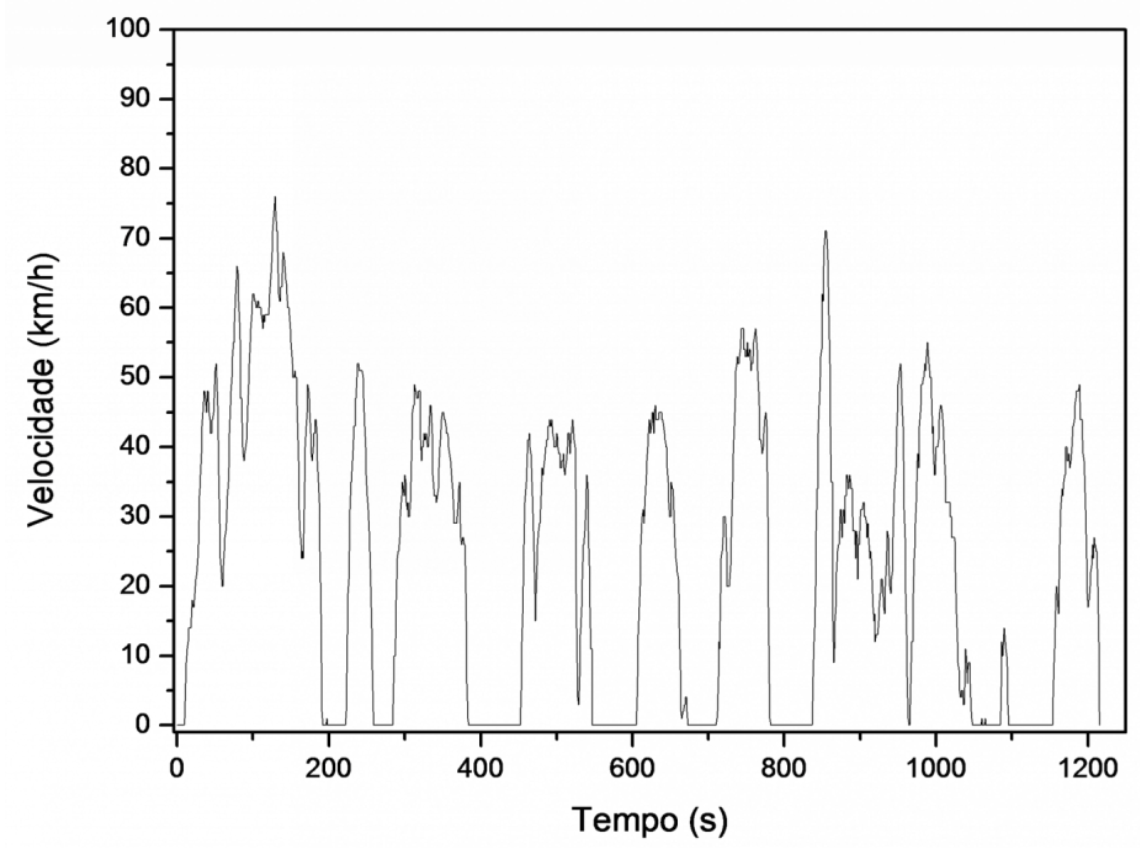

Figura 4. Ciclo de condução urbano típico do veículo leve na cidade de Fortaleza

Como se pode observar na Figura 4, o trajeto é caracterizado pelo tráfego urbano intenso, com sucessivas paradas e retomadas - do inglês stop and go - no qual 43,47\% do tempo o veículo permaneceu no modo de operação idle, $29,66 \%$ no modo de operação aceleração e $26,87 \%$ no modo desaceleração. 0 modo de operação cruzeiro não foi observado durante os testes. A Figura 5 apresenta os perfis de aceleração obtidos durante os experimentos.

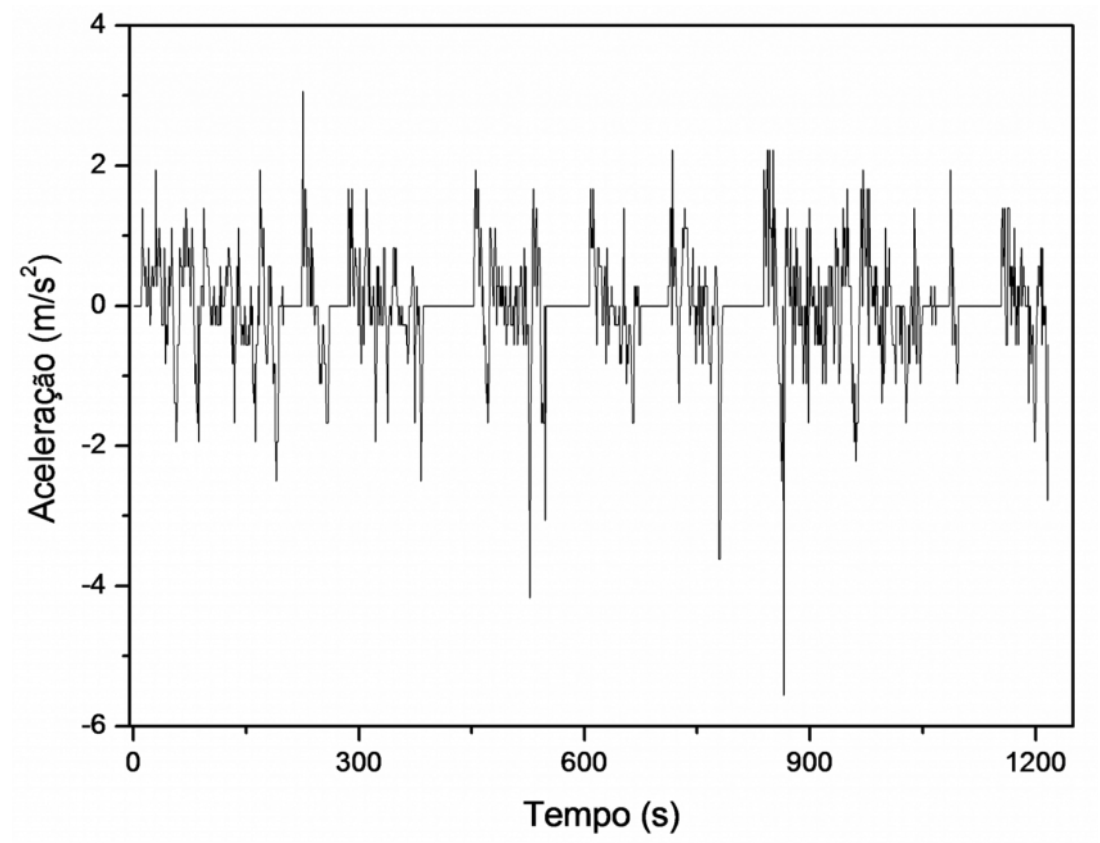

Figura 5. Perfil aceleração instantânea para o veículo leve em um trecho urbano da cidade de Fortaleza

O perfil de aceleração é inerente às condições de tráfego da região, uma vez que o comportamento intermitente desta variável é consequência do stop and go, como citado anteriormente. Esses eventos de curto prazo são responsáveis pelos maiores níveis de emissão, devido tanto ao maior consumo de combustível, quanto à menor entrada de ar na câmara de combustão, fazendo com que a combustão 
seja incompleta e, assim, aumentando as taxas de emissão veicular. 0 perfil de emissões instantâneas de $\mathrm{CO}_{2}$, o consumo de combustível e velocidade estão apresentados na Figura 6 a seguir.

Para o poluente $\mathrm{CO}_{2}$, a maior taxa de emissão média foi observada durante o modo de operação aceleração $(6,38 \mathrm{mg} / \mathrm{s})$, seguido dos modos idle $(4,70 \mathrm{mg} / \mathrm{s})$ e desaceleração $(3,29 \mathrm{mg} / \mathrm{s})$. Em geral, o fator de emissão médio para o $\mathrm{CO}_{2}$ foi de $7,56 \mathrm{mg} / \mathrm{km}$ durante os testes. Os maiores níveis de $\mathrm{CO}_{2}$ são observados quando há um aumento da velocidade, já que a rotação do motor e o consumo de combustível são maiores, como mostram as Figuras 6 e 7.

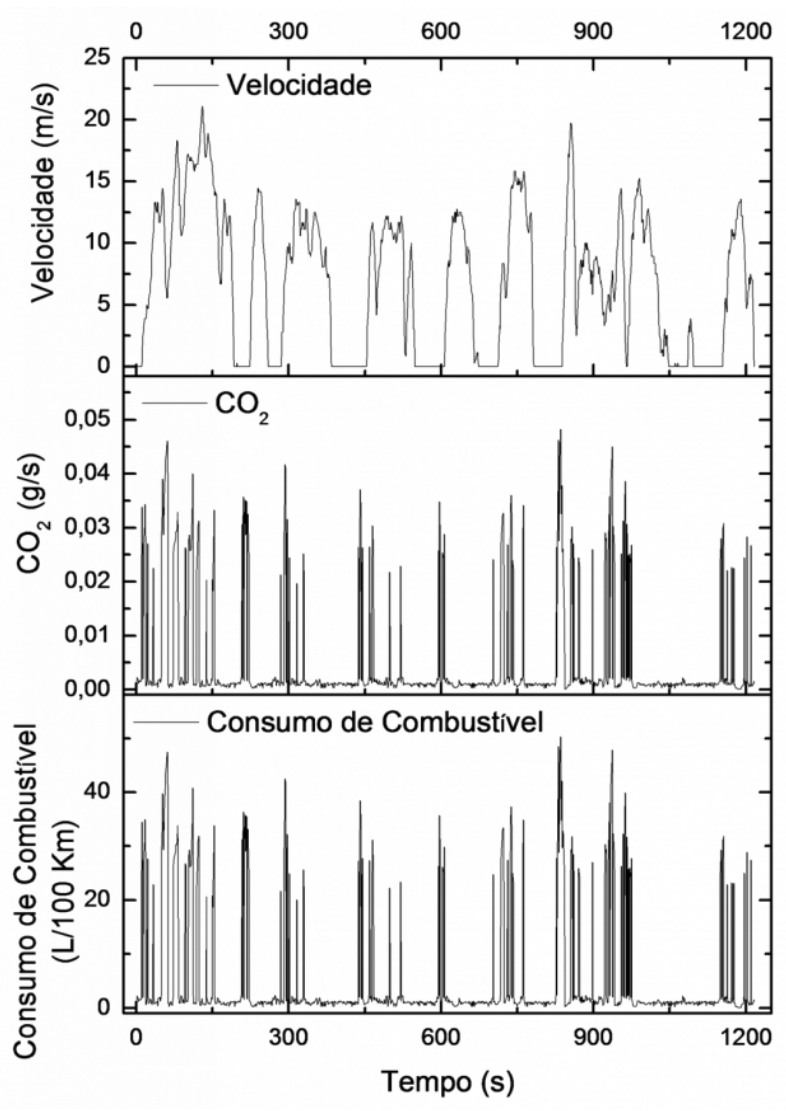

Figura 6. Perfil de velocidade, emissão de $\mathrm{CO} 2$ e consumo de combustível instantâneos para o veículo leve em um trecho urbano da cidade de Fortaleza

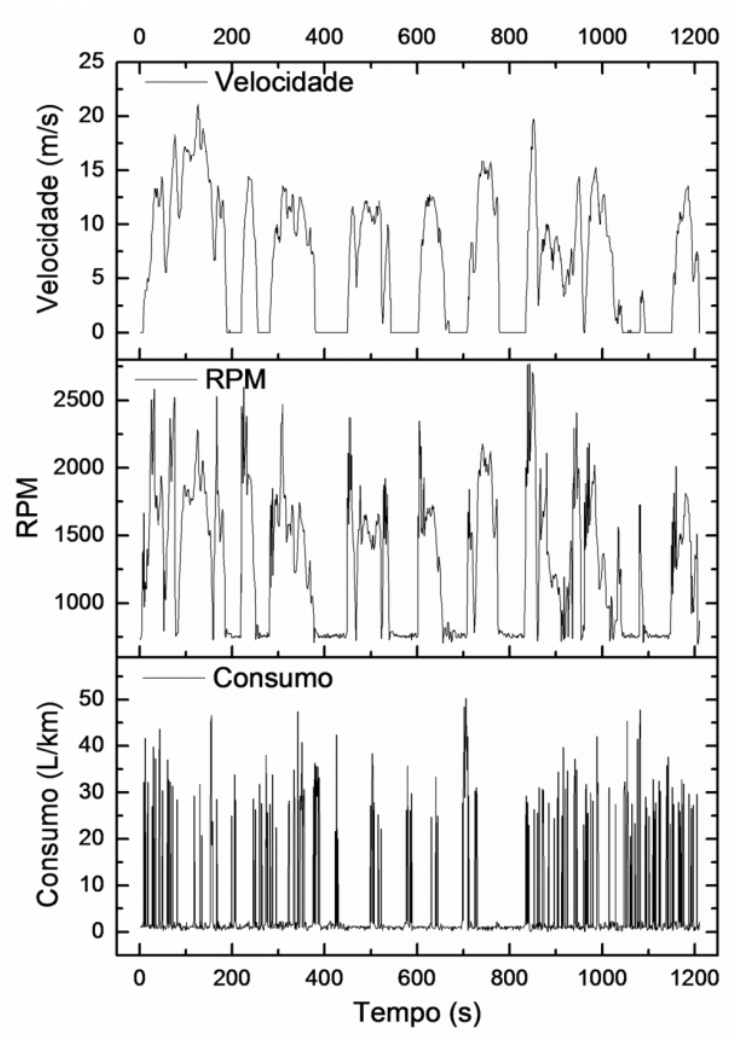

Figura 7. Perfil de velocidade, RPM e consumo de combustível instantâneos para o veículo leve em um trecho urbano da cidade de Fortaleza

Apesar disso, vale ressaltar que em processos de combustão, com a manutenção regular do motor, são observados maiores níveis de $\mathrm{CO}_{2}$, já que quanto mais eficiente é o processo de combustão (maior conversão em $\mathrm{CO}_{2}$ ), mais significativa é a presença deste poluente. Já a diminuição de $\mathrm{CO}_{2}$ pode estar associada ao aumento da emissão de hidrocarbonetos e $\mathrm{CO}$. Assim, a diminuição de $\mathrm{CO}_{2}$ necessariamente faz com que aumente a produção de outros poluentes até bem mais nocivos e de efeitos locais, e consequentemente, os seus efeitos na saúde e meio ambiente.

O carbono presente na composição do combustível é emitido principalmente na forma de $\mathrm{CO}_{2}$, estando assim intimamente correlacionado com o consumo de combustível. Nestes experimentos, foi encontrada uma correlação de $99,8 \%$ entre o consumo de combustível e $\mathrm{CO}_{2}$ emitido, resultado este que corrobora com os observados por Frey et al. (2003), como mostra a Figura 8.

O consumo médio de combustível no modo de operação aceleração foi de 6,61 L/100 km durante os testes, seguido dos modos idle e desaceleração, com 4,84 e 3,39 L/100 km, respectivamente. 0 perfil de emissões de $\mathrm{CO}, \mathrm{NO}_{\mathrm{x}}$, temperatura do gás e rotações por minuto do motor estão apresentados na Figura 9. 


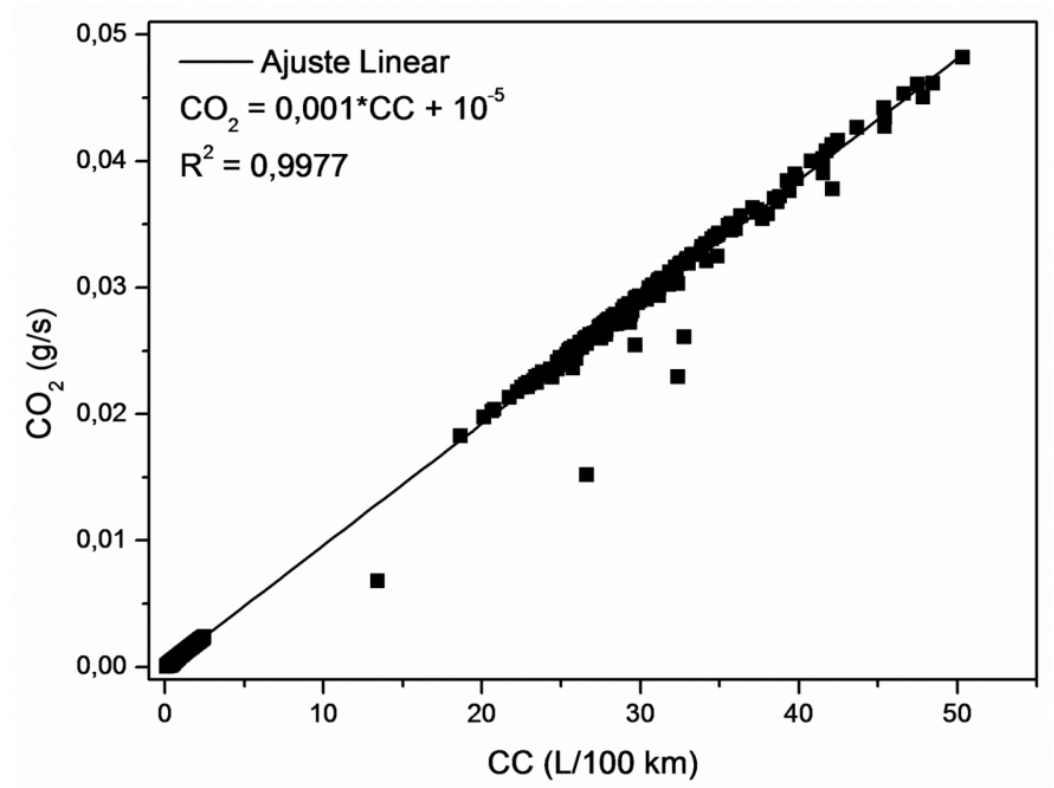

Figura 8. Correlação entre o consumo de combustível e a emissão de $\mathrm{CO}_{2}$ para o veículo leve em um trecho urbano da cidade de Fortaleza

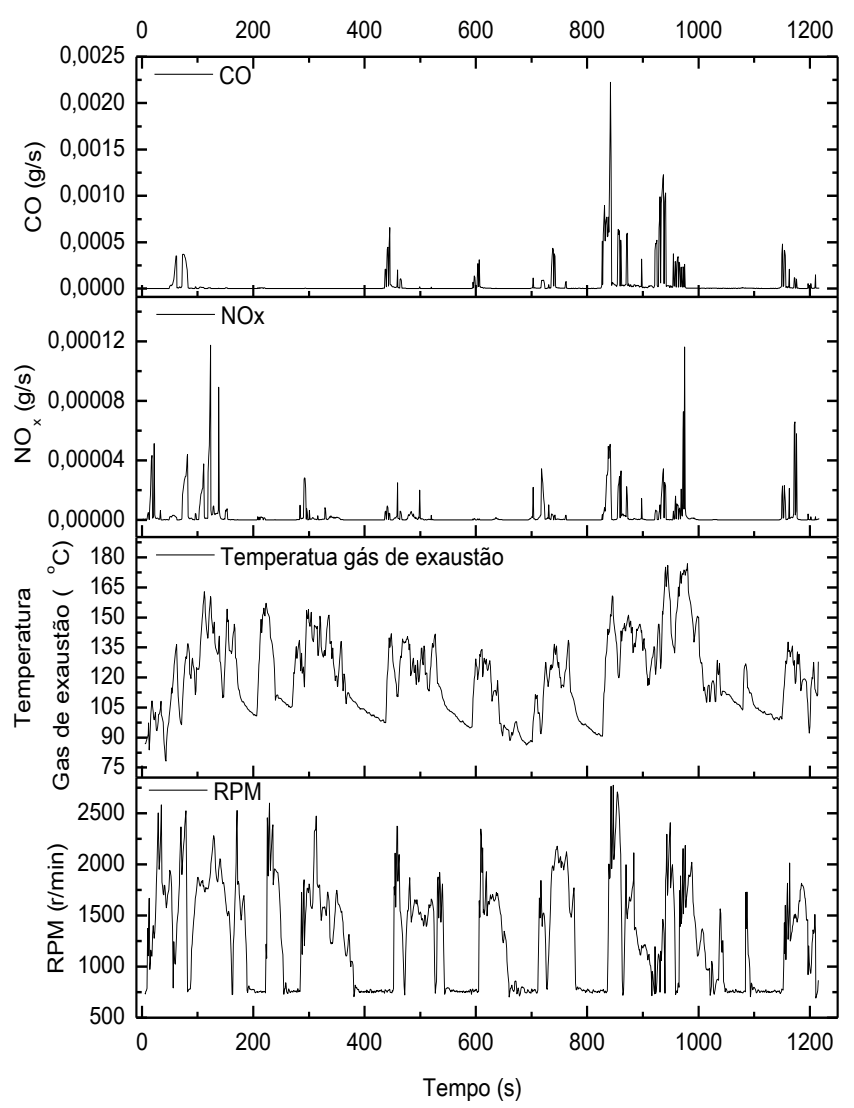

Figura 9. Perfil de emissões de $\mathrm{CO}$ e NO , temperatura do gás e rotações por minuto do motor (RPM) para o veículo leve em um trecho urbano da cidade de Fortaleza

Para o poluente CO, a maior taxa de emissão média foi observada durante o modo de operação aceleração (55,60 $\mu \mathrm{g} / \mathrm{s})$, seguido dos modos idle $(35,01 \mu \mathrm{g} / \mathrm{s})$ e desaceleração $(24,57 \mu \mathrm{g} / \mathrm{s})$. Durante os testes, o fator de emissão médio foi de $6,69 \mu \mathrm{g} / \mathrm{km}$ para o CO. Os maiores níveis de emissão deste poluente foram observados quando a carga do motor requerida foi maior. 
Para o poluente $\mathrm{NO}_{x}$, maiores taxas de emissão foram observados no modo de operação aceleração, com 5,08 $\mu \mathrm{g} / \mathrm{km}$. Para os modos idle e desaceleração as taxas de emissão média foram de 1,98 $\mu \mathrm{g} / \mathrm{km}$ e $1,78 \mu \mathrm{g} / \mathrm{km}$, respectivamente. Durante os testes, o fator de emissão médio foi de $0,44 \mu \mathrm{g} / \mathrm{km}$ para o $\mathrm{NO}_{\mathrm{x}} \mathrm{O} \mathrm{NO}_{\mathrm{x}}$ proveniente da combustão é decorrente do aumento da carga do motor, quando a temperatura tanto da câmara de combustão quanto do gás de exaustão são maiores. Juntamente com as elevadas temperaturas o tempo de residência do gás de combustão são os principais fatores que contribuem para o processo de formação de $\mathrm{NO}_{\mathrm{x}}$ térmico, cujo mecanismo de reações formador do $\mathrm{NO}_{\mathrm{x}}$ térmico foi primeiramente proposto por Zeldovich e Fenimore (MARTINS, 2005). Além disso, em baixas velocidades, as emissões de NO são menores, devido a boa parte dessas emissões se originarem no ar de admissão, que diminui a entrada em menores velocidades. É importante lembrar que a maior emissão de NO se deve ao nitrogênio presente no ar de admissão e não no nitrogênio do combustível, como mostra a Figura 10.

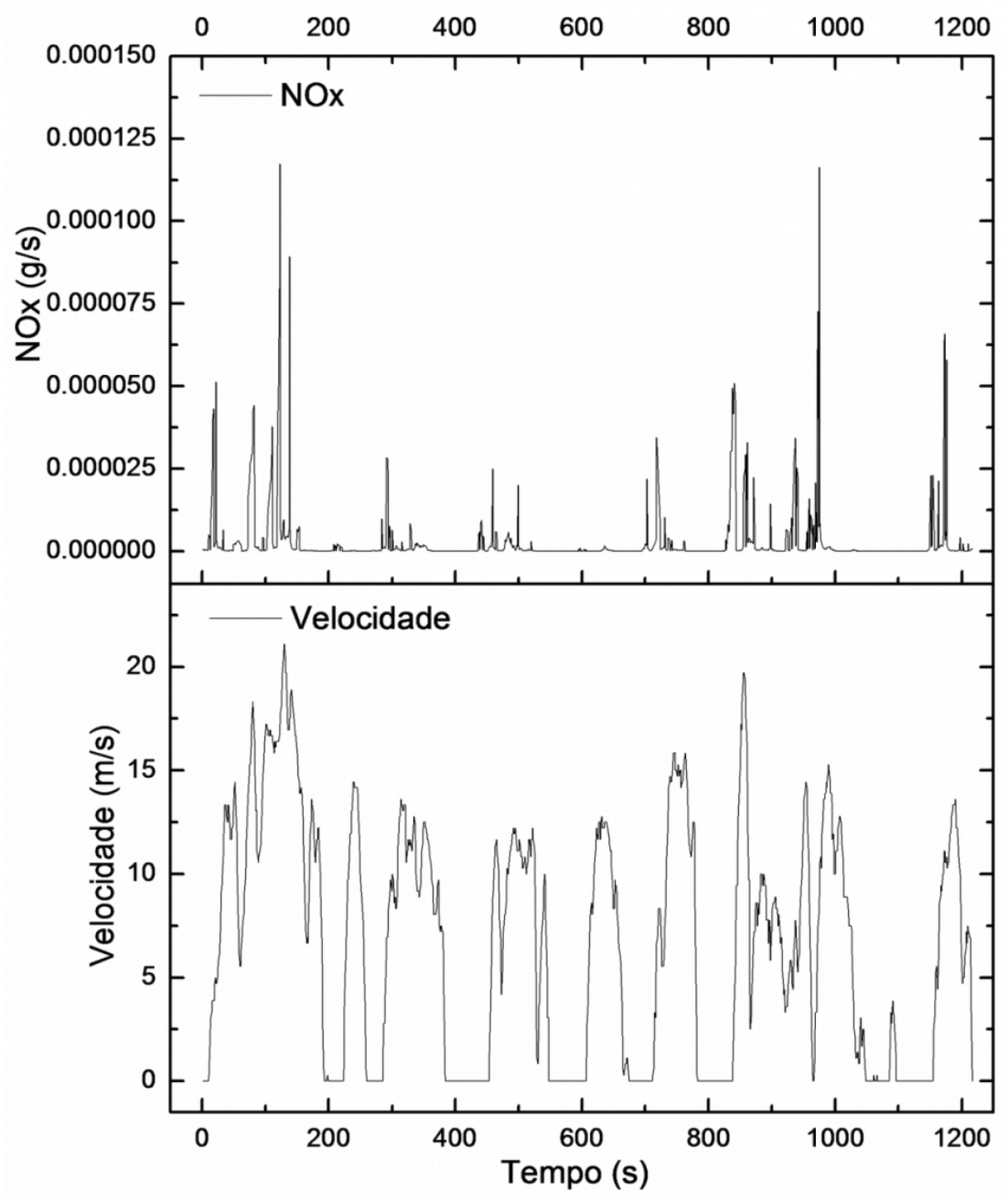

Figura 10. Perfil de emissões de $\mathrm{NO}_{x}$ e velocidade para o veículo leve em um trecho urbano da cidade de Fortaleza

Os níveis observados de hidrocarbonetos durante os testes foram pontuais, especificamente quando um pico elevado de CO foi registrado. A presença de HC praticamente nula durante os testes pode ser atribuída à presença do catalisador, que oxida este poluente. Entretanto, quando a carga do motor é elevada, a fração passante no catalisador tem um baixo tempo de residência, fazendo com que o tratamento dos gases não seja efetivo e a presença de HC e CO é detectada nos gases de exaustão devido a combustão incompleta, como pode ser observado na Figura 11. 

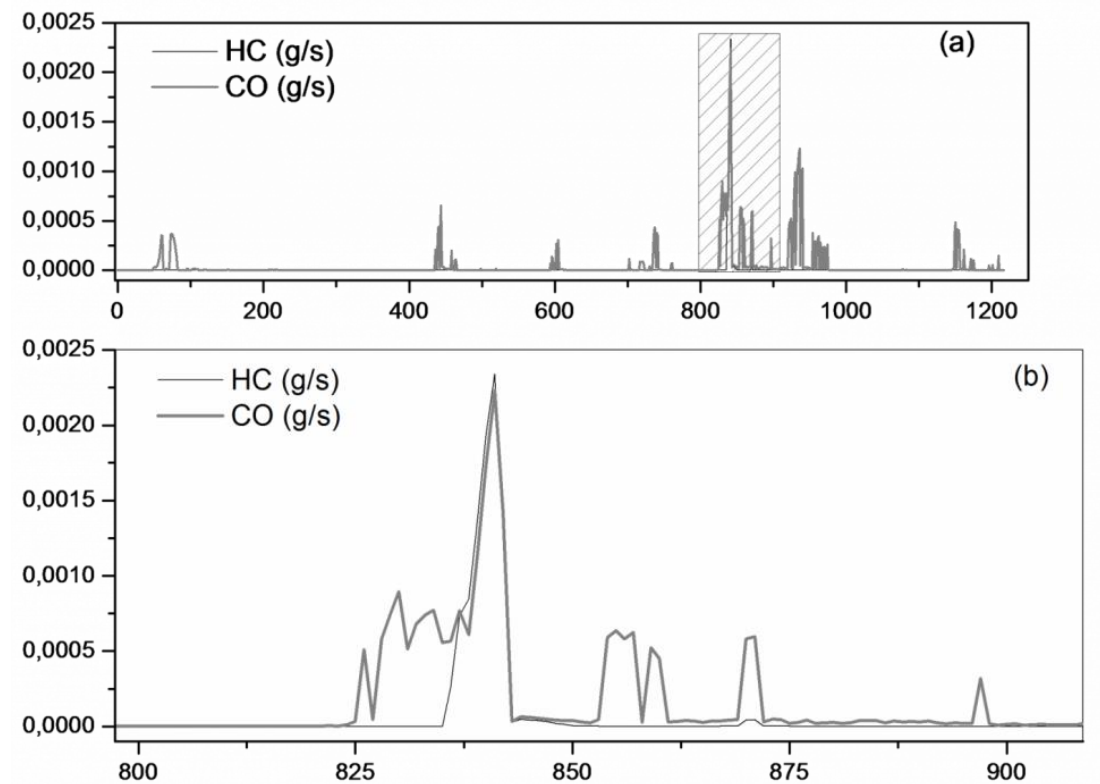

Figura 11. (a), Perfis de emissões de HC e CO para o veículo leve flex em um trecho urbano da cidade de Fortaleza, (b) Pico de $\mathrm{HC}$ detectado durante os testes

Em relação ao $\mathrm{SO}_{2}$, não foi detectada a presença deste poluente durante os testes, o que pode ser atribuída à composição do combustível, que tem limite máximo de 50 ppm de enxofre, conforme legislação brasileira (ANP, 2015). De forma resumida, a Figura 12 mostra como o modo de operação do motor do veículo teve impacto significativo no consumo de combustível, e, consequentemente, nas emissões de poluentes.

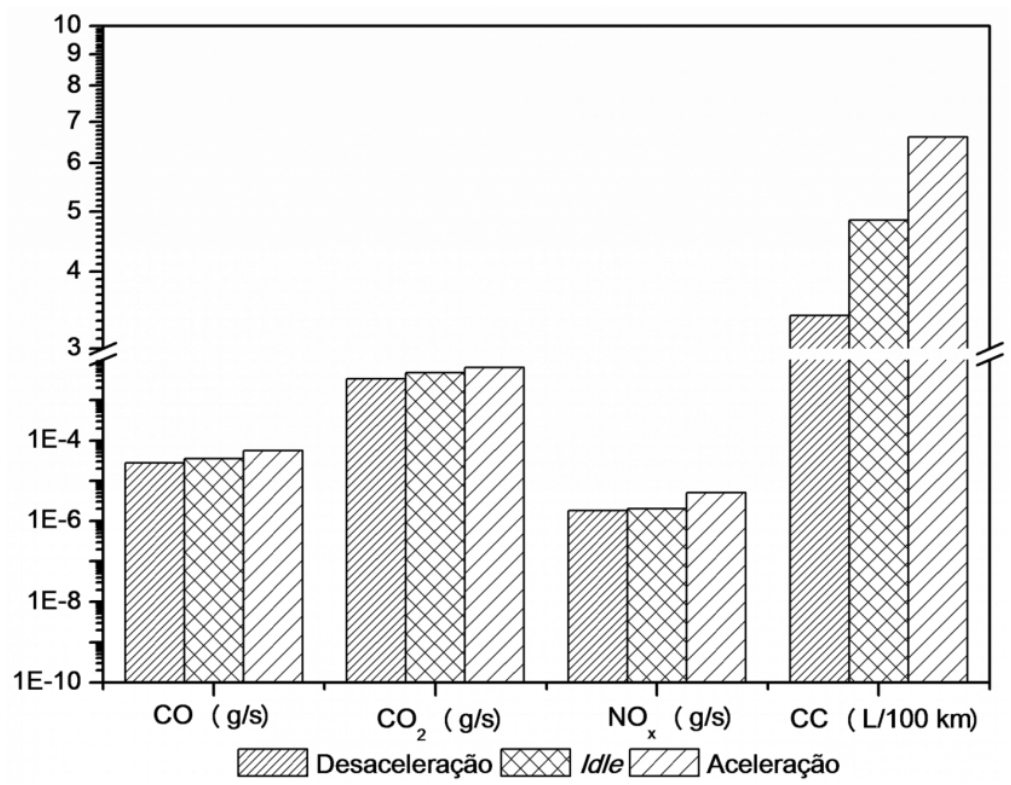

Figura 12. Influência dos modos de operação nas emissões de $\mathrm{CO}, \mathrm{CO}_{2}, \mathrm{NO}_{\mathrm{x}}$ e consumo de combustível para o veículo leve em um trecho urbano da cidade de Fortaleza

De acordo com a Figura 10, além do consumo de combustível, o modo de operação aceleração também foi responsável pela maior representatividade dos poluentes $\mathrm{CO}_{2}$, $\mathrm{CO}$ e $\mathrm{NO}_{\mathrm{x}}$ emitidos, como citado anteriormente. 0 modo de operação desaceleração teve menor impacto no consumo de combustível e nas emissões de poluentes, em função da menor potência requerida do motor. 
A condição de tráfego na região de estudo, caracterizada pelo stop and go, caracterizado por grandes flutuações de velocidade, faz com que as taxas de emissões sejam maiores que no tráfego em rodovias. Apesar de não ter sido observado durante os testes, o modo de operação cruzeiro, por ser caracterizado por velocidades constantes e aceleração nula, a emissão de poluentes é menor, já que é injetada uma menor quantidade de combustível. Nesse contexto, a fluidez do tráfego em áreas urbanas é favorável a menores taxas de emissão.

\section{CCONCLUSÃO}

Um ciclo de condução foi construído para um trecho urbano da cidade de Fortaleza e foi caracterizado pelo tráfego intenso, com sucessivas paradas e partidas (stop and go). Esses eventos de curto prazo favoreceram um maior consumo de combustível e, consequentemente, às emissões dos poluentes medidos. Dentre os modos de operação analisados, observou-se um maior impacto do modo aceleração nas emissões, apesar do modo idle ter representado mais de $40 \%$ do tempo.

Destaca-se que este trabalho coletou tanto as emissões instantâneas de poluentes durante os testes, quanto os parâmetros necessários para a construção do ciclo real de condução, além de parâmetros do motor e características de via. Em geral, os trabalhos realizados estimam as emissões através de ciclos de condução sintéticos, ou seja, realizados em dinamômetros, e não conseguem captar os eventos de curto prazo, inerentes ao tráfego urbano, como demonstrado neste trabalho.

Em geral, foi possível observar que o modo de operação aceleração é responsável por maiores taxas de emissões dos poluentes $\mathrm{CO}_{2}, \mathrm{CO}$ e $\mathrm{NO}_{\mathrm{x}}$ e consumo de combustível, sendo atribuído aos eventos transitórios (stop and go), inerentes às características do tráfego local. Não foram observados durante os testes o modo de operação cruzeiro, já que não foram observadas velocidades constantes, em função da dinâmica do tráfego local. Sugere-se que as emissões e os ciclos de condução sejam avaliados em outros tipos de via da região urbana, bem como a influência do teor de etanol presente da gasolina brasileira.

\section{REFERÊNCIAS}

Abreu, A. A.; Ribeiro S. K. (2003). O potencial de redução das emissões de monóxido de carbono, através da implantação de programas de inspeção e manutenção: o caso do Estado do Rio de Janeiro. In: XVII CONGRESSO DE PESQUISA E ENSINO EM TRANSPORTES, 17., 2003. Anais... Rio de Janeiro: ANPET. p. 375-387,

Associação o Brasileira de Normas Técnicas (2002). NBR7024: veículos rodovia rios automotores leves: medição do consumo de combustível. Rio de Janeiro, RJ: ABNT.

Associação Brasileira de Normas Técnicas (2012). NBR6601: veículos rodoviários automotores leves: determinação de hidrocarbonetos, monóxido de carbono, óxidos de nitrogênio, dióxido de carbono e material particulado no gás de escapamento. Rio de Janeiro, RJ: ABNT.

Associação Brasileira de Normas Técnicas (2015). NBR13992: gasolina automotiva: determinação do teor de álcool etílico anidro combustível (AEAC). Rio de Janeiro, RJ: ABNT.

Agência Nacional do Petróleo, Gás Natural e Biocombustíveis (2015). Legislação. Disponível em: <http://www.anp.gov.br/wwwanp/legislacao>. Acesso em: 09 out. 2015.

Brasil (2015). Portaria no 75, de 5 de março de 2015: fixa o percentual obrigatório de adição de etanol anidro combustível à gasolina. Diário Oficial da União, Brasília, DF, n. 44, p. 17, 6 mar. Seção I.

Cappiello, A. (2002). Modeling traffic flow emissions. Massachussets: Department of Civil and Environmental Engineering.

Cassiano, D. R.; Azevedo, J. A. H.; Dias, H. L. F. ; Araujo, R. S. ; Cavalcante, F. S. A.; Bertoncini, B. V. ; Policarpo, N. A. ; Oliveira, M. L. M. (2016) MAMUTE: Monitoring AutoMotive Unit Transit Emissions Development and Application for Flex-fuel and Diesel Vehicles. In: Markus Helfert, Oleg Gusikhin. (Org.). INTERNATIONAL CONFERENCE ON VEHICLE TECHNOLOGY AND INTELLIGENT TRANSPORT SYSTEMS, 2., 2016. Anais.... .Portugal: SCITEPRESS Science and Technology Publications, Lda., v. 1, p. 237-244.

Departamento Nacional de Trânsito - DENATRAN (2016). Frota por município. Disponivel em: <http://www.denatran.gov.br/index.php/estatistica/261-frota-2016> Acesso em: 09 out. 2016.

Dias, H. L. F., Bertoncini, B. V., Oliveira, M. L. M, Cavalcante, F. S. A., Araújo, R. S. (2015). Procedimento para coleta dinâmica embarcada de emissões provenientes de veículos transportadores de carga em área urbana. Transportes, v. 23, n.3, p. 1825. DOI: $10.14295 /$ transportes.v23i3.888.

Frey, H. C.; Unal, A.; Rouphail, N. M.; Colyar, J. D. (2003). On-road measurement of vehicle tailpipe emissions using a portable instrument. Journal of the Air \& Waste Management Association, v. 53, n. 8, p. 992-1002.

DOI: $10.1080 / 10473289.2003 .10466245$. 
International Agency for Research on Cancer - IARC (2013). IARC monographs on the evaluation of carcinogenic risks to humans. v. 109. Outdoor air pollution.

Instituto Brasileiro de Geografia e Estatística - IBGE. (2016). Cidades@. Disponível em: <http://www.cidades.ibge.gov.br/xtras/home.php>. Acesso em: 09 out. 2016.

Lin, C. A.; Pereira, L. A. A.; Conceição, G. M. S.; Kishi, H. S.; Milani, R.; Braga, A. L. F.; Saldiva, P. H. N. (2003). Association between air pollution and ischemic cardiovascular emergency room visits. Enviromental Research, v. 92, p. 57-63.

DOI: 10.1016/S0013-9351(02)00054-3.

Martins, J. (2005). Motores de combustão interna. Porto: Publindústria,

Pelkmans, L.; Debal, P. (2006). Comparison of on-road emissions with emissions measured on chassis dynamometer test cycles. Transportation Research Part D, v. 11, p. 233-241. DOI: 10.1016/j.trd.2006.04.001.

Programa de Controle de Poluição do Ar por Veículos Automotores - PROCONVE (2011). Manual PROCONVE/PROMOT. 3. ed. Coleção Meio Ambiente. Série Diretrizes-Gestão n. 3. Brasília: IBAMA.

Vaz De Melo, C. R. (2005). Desenvolvimento de uma metodologia para determinar a concentração dos gases emitidos pelos veículos automotores leves de ciclo otto em condições reais de operação. Dissertação (Mestrado) - Universidade de Brasília, Brasília, Distrito Federal.

Xiao, Z.; Dui-Jia, Z.; Jun-Min, S. (2012). A synthesis of methodologies and practices for developing driving cycles: energy procedia. International Conference on Future Energy, Environment, and Materials Part C, v. 16, p. 1868-1873. DOI: 10.1016/j.egypro.2012.01.286.

Zhen, X.; Wang, Y. Numerical analysis on original emissions for a spark ignition metanol engine based on detailed chemical kinetics. Renewable Energy, n. 81, p. 43-51, 2015. DOI: 10.1016/j.renene.2015.03.027. 